\title{
Resistencias locales, el caso de la prohibición de instalación de antenas de telefonía celular en diálogo con estrategias de gestión de riesgos ${ }^{1}$
}

María Valeria Berros ${ }^{2}$

\section{Resumen}

Uno de los mecanismos a través de los cuales se gestionan riesgos relativos al medioambiente y la salud humana es el establecimiento de mínimos de tolerancia o estándares, constituyéndose como una de las tecnologías jurídicas que dan cuenta de la emergencia, expansión y desarrollo de la racionalidad precautoria.En el caso de la determinación de límites de emisión de radiaciones no ionizantes provenientes de la instalación de antenas de telefonía celular, se observa esta estrategia globalizada de gestión de riesgos que puede pensarse a modo de contribución en el debate acerca del proceso de globalización en el campo del derecho.Partimos de esta tesis, para recrear, ahora, cuales son las estrategias que localmente se construyen en "contrapunto" con esta estrategia. Para ello, se efectúa el relevamiento de un caso puntual y un recorrido sobre las herramientas jurídicas articuladas a los fines de plantear resistencias a la instalación de estos agentes de emisión, aún en los supuestos en que cumplimenten con los límites de emisión legalmente autorizados.

Palabras-claves: Riesgos. Resistencia. Racionalidad precautoria.

1 Este artigo está cadastrado no Digital Object Identifier System sob o número doi: 10.5102/ prismas.2010.07.1.01 Disponível em: <www.publicacoesacademicas.uniceub.br $>$.

2 Abogada Especialista en Derecho de Daños FCJS - UNL. Jefa de Trabajos Prácticos Ordinaria en "Derecho Civil II" y Ayudante de Cátedra en la asignatura "Introducción a la Sociología", FCJS, UNL. Becaria Doctoral CONICET - UNL. Miembro del Equipo de investigaciones en "Bioregulaciones" dirigido por Gonzalo Sozzo del Centro de Investigaciones de la Facultad de Ciencias Jurídicas y Sociales, Universidad Nacional del Litoral. 


\section{Introducción}

En un trabajo anterior ${ }^{3}$, se analizó la determinación de estándares focalizando en su carácter de tecnología jurídica para la gestión de riesgos generados por la emisión de radiaciones no ionizantes ${ }^{4}$ - específicamente devenidos de la instalación de antenas de telefonía móvil en Argentina - como una manifestación del proceso de globalización del derecho.

Se considera esta herramienta jurídica dentro del marco de la emergencia de la racionalidad precautoria. Ésta se problematiza desde la teoría social hacia fines del siglo XX e inicios del presente, indagando particularmente en la proliferación de "híbridos", riesgos "manufacturados"6 a los que subyace la incerteza o controversia científica. En el campo del derecho se da cuenta de esta racionalidad en la serie de tecnologías jurídicas que la traducen.

El tema que aquí nos ocupa, se inserta en un contexto en el que el conocimiento científico disponible no puede explicar acabadamente las posibles consecuencias dañosas que generaría a la salud humana y el medioambiente la exposición de modo continuado a radiaciones no ionizantes aún por debajo de los topes permitidos. Existen grupos expertos organizados ${ }^{7}$ y equipos de investigación diseminados en diferentes pa-

3 BERROS, Maria Valeria. La estandarización como tecnología precautoria globalizada: el caso de las radiaciones no ionizante. Prismas: direito, políticas públicas e mundializaçao, Brasilia, v. 5, n. 2, p. 151-186, jul./dez. 2008. Colección Universitaria UniCEUB. ISSN 1908-3680.

4 La Organización Mundial de la Salud explica que "Las radiaciones compuestas por cuantos de luz sin energía suficiente para romper los enlaces moleculares se conocen como «radiación no ionizante». Las fuentes de campos electromagnéticos generadas por el hombre que constituyen una parte fundamental de las sociedades industriales (la electricidad, las microondas y los campos de radiofrecuencia) están en el extremo del espectro electromagnético correspondiente a longitudes de onda relativamente largas y frecuencias bajas y sus cuantos no son capaces de romper enlaces químicos [...]”. ORGANIZACIÓN MUNDIAL DE LA SALUD. ¿Qué son los campos electromagnéticos? Disponible en: <www.who.int/peh-emf/es/>. Acceso en: Junio 2009.

5 LATOUR, Bruno. Nous navons jamais été moderne. Paris: La Decouverte, 1997.

6 BECK, Ulrich. La sociedad del riesgo mundia. Espana: Paidós, 2007.

7 Se destaca el programa interdisciplinario denominado "Projet Internacional CEM" Este proyecto fue establecido en el año 1996 por la OMS, dirigido por Michael Repacholi. El mismo no sólo pretende hacer avanzar las investigaciones científicas, sino también formular recomendaciones para las autoridades nacionales respecto de la gestión de riesgos provenientes de la generación de campos electromagnéticos. A este objetivo, se suma también el de informar al público, lo cual se concretiza en la publicación regular de memorias y, por ejemplo, en la confección del documento citado en el presente trabajo: "Estableciendo un diálogo sobre los campos electromagnéticos". 
íses que se encuentran estudiando el problema, y sus conclusiones no son homogéneas. Se trata de una hipótesis de hecho que "posiblemente" podría generar algún tipo de perjuicio, pero no existe uniformidad en los discursos de quienes conforman la "comunidad experta". Asimismo, no todos los estudios siguen la misma metodología en su estructuración y desarrollo. Por ello se presentan dificultades en su cotejo o comparación e, incluso, los criterios que se toman en consideración para determinar la adecuación o no de las investigaciones en la materia pueden ser objeto de problematización.

Por su parte, desde el derecho "se aportan" tecnologías jurídicas a los fines de coadyuvar a gobernar este riesgo. Una de las más relevantes es la fijación de "estándares" o topes de emisión". La construcción de esta "estrategia de estandarización" permitiría una "normalización del riesgo". Normalización construida a partir de una suerte de "alianza" que podría ser pensada en clave de ratificación de los discursos del expertise científico.

A los fines de estudiar la problemática referenciada, reconstruyendo algunas articulaciones entre "lo global" y "lo local", se indagó el modo mediante el cual se visibiliza el traslado de normas y sus racionalidades subyacentes, desde las agencias globales hacia los estados nacionales, focalizándose en la observación de la legislación interna así como, también, en los decisorios jurisprudenciales argentinos. Para ello, se partió de la perspectiva de "espacios jurídicos diferenciados" de Boaventura de Sousa Santos, quien distingue los espacios internacional, nacional y local y las tres formas de derecho que se corresponden a cada uno de ellos: el derecho global, nacional y local. Estas formas de derecho son explicadas en función del tamaño de la "escala" mediante la cual regulan las acciones sociales. El derecho local se configura como una legalidad a "gran escala", el derecho nacional como la "escala media" y el derecho global como la "pequeña escala"9.

Desde esta perspectiva teórica se estudiaron los ensamblajes entre las escalas jurídicas descriptas, considerando cómo se pautan los topes de emisión de

\footnotetext{
8 Se destacan, también, otras tecnologías jurídicas abocadas al gobierno de este riesgo como, por ejemplo, las evaluaciones de impacto ambiental, cuya presentación es un requisito obligatorio para la instalación de antenas de telefonía celular por ejemplo en el caso de la Provincia de Santa Fe tanto la legislación provincial (Ley n²12632/04) como la local (Ordenanza $\mathrm{N}^{\circ} 10578$ de la ciudad de Santa Fe) así lo exigen.

9 SANTOS, Boaventura de Sousa. Crítica de la razón indolente: contra el desperdicio de la experiencia. Bilbao: Desclée de Brouwer, 2003.
} 
radiaciones no ionizantes, específicamente, escrutando el caso de la instalación de antenas de telefonía móvil ${ }^{10}$.

${ }^{10}$ Se tomó como punto de partida el análisis de la normativa que regula la materia y el conjunto de casos jurisprudenciales sobre esta problemática en: ARGENTINA. Cámara Civil y Comercial de Santa Fé. Acción de amparo. Demanda, contestación de demanda, sentencia de primera instancia y sentencia de la Cámara Civil y Comercial de Santa Fe, Sala 2.Farina, Pablo M. c/ Compañía de Radiocomunicaciones Móviles S.A. s/ Acción de amparo (Zeuz, Tomo 89 - J 360 y siguientes). Santa Fé, 4 de mayo de 2001. ARGENTINA. Tribunal Superior de Córdoba. Sentencia de Castellani, Carlos y E. y otros (Responsabilidad Civil y Seguros). Buenos Aires, 11 de março de 2003. v. 6, n. 6, nov./dic. 2003. ARGENTINA. Cámara Federal de Apelaciones de La Plata. Sentencia de la Causa 3801/02 de La, Sala II, Asociación Coordinadora de Usuarios, Consumidores y Contribuyentes c/ ENRE - EDESUR s/ cese de obra de cableado y traslado de Subestación Transformadora. Córdoba, 8 de agosto de 2003. Disponíble en: <www.lexisnexis.com.ar $>$ Acceso en: abril 2009 ARGENTINA. Cámara de Apelaciones en lo Civil y Comercial de Rosário. Sentencia de la Cámara de Apelaciones en lo Civil y Comercial de Rosario - Sala I, Vecinos de la Ciudad de Rosario c/ Municipalidad de Rosario. Rosário, 05 de octubre de 2004. LLLitoral Abril 2005, 298. ARGENTINA. Cámara de Apelaciones en lo Civil y Comercial de Rosário. Sentencia de la Cámara de Apelaciones en lo Civil y Comercial de Rosario Sala I, Vecinos de la Ciudad de Rosario c/ Municipalidad de Rosário. Rosário, 26 de diciembre de 2005. LLLitoral Mayo 2006, 524. ARGENTINA. Medida Cautelar. Sentencia En primera instancia del Juzgado en lo Contencioso, Administrativo y Tributario Número 12 de la Ciudad Autónoma de Buenos Aires. "Peino, Leonardo Esteban y otros contra GCBA sobre amparo (art.14 CCABA). Buenos Aires, 18 de octubre de 2006. Disponible en: <www.diariojudicial.com.ar>. Acceso en: marzo 2009 ARGENTINA. Sentencia en causa 17008/03 del Juzgado de Primera Instancia en lo Civil $\mathrm{N}^{\circ} 105$ de Buenos Aires. Espíndola María c/ Movicom Bellsouth s/ Daños Perjuicios. Buenos Aires, 09 de febrero de 2007. Disponible en: <www.diariojudicial.com.ar>. Acceso en: marzo 2009 ARGENTINA. Sentencia de la Cámara en lo Civil y Comercial de la ciudad de Córdoba. no 5, Asís, María F. c/ Giorda Mario y otro s/ daño temido. Córdoba, 20 de marzo de 2007. Disponible en: $<\underline{w w w}$. lexisnexisonline> Acceso en: abril 2009 ARGENTINA. Cámara Federal de Apelaciones de La Plata. Sentencia de la Cámara Federal de Apelaciones de La Plata, Sala I, Telefónica Comunicaciones Personales SA c/ Municipalidad de Lanús s/ acción declarativa de insconstitucionalidad. La Plata, 28 de junio de 2007. Disponible en: <www.eldiariojudicial.com.ar >. Acceso en: marzo 2009 ARGENTINA. Sentencia del Juzgado en lo Contencioso, Administrativo y Tributario No 12 de la Ciudad Autónoma de Buenos Aires, Uyemas Héctor c/ Gobierno de la Ciudad Autónoma de Buenos Aires s/ amparo. Buenos Aires, 18 de septiembre de 2007. Disponible en: <www. eldiariojudicial.com,.ar>. Acceso en: marzo 2009 ARGENTINA. Sentencia de la Cámara Federal de Apelaciones de La Plata - Sala III, Agüero, Norberto y otros c/ Municipalidad de Cañuelas s/ amparo. La Plata, 25 de octubre de 2007. Disponible em: <www.eldiariojudicial.com.ar $>$. Acceso en: marzo 2009 ARGENTINA. Sentencia de la Cámara de Apelaciones en lo Contencioso, Administrativo y Tributario - Sala II, Gallo, Susana Beatriz c/ GCBA s/ Amparo (art. 14 CCABA) Causa N ${ }^{\circ}$ 25208/0. Ciudad Autónoma de Buenos Aires, 22 de noviembre de 2007. Disponible en: $<$ www.eldiariojudicial.com.ar> . Acceso en: marzo 2009 ARGENTINA. Sentencia de la Cámara Nacional de Apelaciones en lo Civil de la Ciudad Autónoma de Buenos Aires - Sala H, Bottero, Enrique y otros c/ Nextel Communications Argentina SA s/ daños y perjuicios. Buenos Aires, 5 de diciembre de 2007. Disponible en: <www.lexisnexisonline.com.ar>. Acceso en abril 2009. 
Prismas: Dir., Pol. Publ. e Mundial., Brasília, v. 7, n. 1, p. 1-20, jan./jun. 2010

Resistencias locales, el caso de la prohibición de instalación de antenas ...

Luego de analizar la forma a través de la cual las normas de la "pequeña escala"11 se expanden hacia la "mediana"12 y "gran"13 escala parecía vislumbrarse que la lógica de la estandarización se extendería de manera lineal desde la esfera internacional a la local.

Sin perjuicio de ello, ya se advertía sobre la existencia de algunas alternativas de "resistencia local"14 que permiten una suerte de "corrimiento" respecto de la lógica de la "estandarización"15.

En el presente trabajo, proponemos explorar una manifestación que es plausible de ser analizada como "resistencia local" a esta "estrategia global" de gestión

${ }^{11}$ Especialmente se trabajó analizando materiales provenientes de la Comisión Internacional para las Radiaciones No - Ionizantes que trabaja en cooperación con la División de Salud Ambiental de la Organización Mundial de la Salud como espacios normativos de carácter no estatal y de raigambre global, que postulan una amplia serie de recomendaciones en cuanto al establecimiento de estándares en esta materia mediante la realización de una serie de documentos entre los que destacamos "Marco para el desarrollo de estándares de CEM (campos electromagnéticos) basados en la salud" y "Legislación modelo para la protección contra campos electromagnéticos”. Disponibles en: $<$ www.who.int $>$. Acceso en: febrero 2009.

${ }^{12}$ Se revisó la normativa nacional: "Estándart Nacional de Seguridad para la exposición a radiofrecuencias comprendidas entre $100 \mathrm{KHz}$ y $300 \mathrm{GHz}$, aprobado por Resolución del Ministerio de Salud y Acción Social n a 202/1995, de conformidad con lo establecido en el "Manual de Estándares de Seguridad para la exposición a radiofrecuencias comprendidas entre $100 \mathrm{KHz}$ y $300 \mathrm{GHz}$ ” "Radiación de radiofrecuencias: consideraciones biofísicas, biomédicas, y criterios para el establecimiento de estándares de exposición”, Volúmenes I y II, respectivamente, de prospección de radiación electromagnética ambiental no ionizante, por medio del que se establecen los límites máximos de irradiación, lo que se constituye de cumplimiento obligatorio según la Resolución n 530/2000 de la Secretaría de Estado de Comunicaciones y la Resolución N 3690/2004 de la Comisión Nacional de Telecomunicaciones. Asimismo, se analizaron publicaciones de la Comisión Nacional de Telecomunicaciones, entre las que se destaca "Radiaciones no ionizantes" de febrero de 2007.

${ }^{13}$ Se estudió la Ley de Telefonía Celular na 12.362/2004 de la provincia de Santa Fe y la Ordenanza Na 10.578/2000 que regula la materia de antenas de telefonía móvil en la ciudad de Santa Fe.

${ }^{14}$ SANTOS, Boaventura de Sousa. A globalizacao e as ciencias sociais. Sao Paulo: Cortez, 2002.

${ }^{15}$ Por ejemplo, en la localidad de Firmat en la Provincia de Santa Fe, "[... Con el propósito de proteger el medio ambiente y la salud de la población, el Concejo Municipal de esta ciudad sancionó una ordenanza que prohíbe la instalación de antenas de telefonía celular dentro del radio urbano. La normativa es similar a la que aplicaron otras localidades santafesinas donde también se intenta evitar posibles contaminaciones electromagnéticas generadas por las radiaciones que emanan estos aparatos [...]" en el artíulo periodístico "Prohiben la instalación de antenas de telefonía celular en Firmat". Diario La Capital de fecha 1 de junio de 2006. 
de riesgos, partiendo del análisis de una de las sentencias judiciales abordadas ${ }^{16}$ que permite introducir una serie relevante de indagaciones en cuanto a las condiciones de posibilidad para resistir "localmente" - utilizando "el derecho" como una herramienta central - frente a la instalación de antenas de telefonía.

En la misma, se debate sobre la viabilidad de la "prohibición local" para la instalación de antenas. Ello supone referir a las competencias que el ámbito municipal posee para el gobierno de riesgos y, más aún, en supuestos en los que éstos son controvertidos y susceptibles de afectar la salud humana y el ambiente. Implica preguntarse sobre la potencialidad de una resistencia construida vía articulación de estrategias jurídicas "locales", en referencia a una estrategia "global".

\section{El primer momento: "clausurar/desactivar". El segundo momento: "Io litigial"}

\subsection{Sobre una prohibición "local" a la instalación de antenas de telefonía móvil.}

La determinación de estándares en Argentina en materia de radiaciones no ionizantes se encuentra regulada por la Resolución 202/1995 que fija el "Estándart Nacional de Seguridad para la exposición a radiofrecuencias comprendidas entre 100 $\mathrm{KHz}$ y $300 \mathrm{GHz}$ ", de conformidad con lo establecido en el "Manual de Estándares de Seguridad para la exposición a radiofrecuencias comprendidas entre $100 \mathrm{KHz}$ y 300 GHz" y "Radiación de radiofrecuencias: consideraciones biofísicas, biomédicas, y criterios para el establecimiento de estándares de exposición", Volúmenes I y II, respectivamente, de prospección de radiación electromagnética ambiental no ionizante ${ }^{17}$.

Esta norma, establece los límites máximos de irradiación que devienen de cumplimiento obligatorio según la Resolución n 530/2000 de la Secretaría de Estado de Comunicaciones.

\footnotetext{
${ }^{16}$ ARGENTINA. Sentencia de la Cámara Federal de Apelaciones de La Plata, sala 1, 28 jun. 2007. Telefónica comunicaciones personales SA c/ Municipalidad de Lanús s/ acción decla-

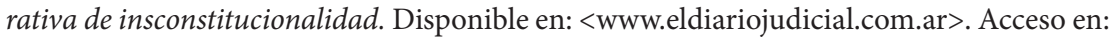
marzo 2009.

${ }^{17}$ Lo expuesto forma parte del contenido de la Resolución n 202/ 1995 del Ministerio de Salud y Acción Social de la Nación.
} 
Prismas: Dir., Pol. Publ. e Mundial., Brasília, v. 7, n. 1, p. 1-20, jan./jun. 2010

Asimismo, la Resolución n³690/2004 de la Comisión Nacional de Telecomunicaciones, establece en su artículo 4 que "[...]Los sistemas irradiantes que no cumplan con los valores máximos establecidos en la Resolución n 202/1995 del Ministerio de Salud y Acción Social de la Nación y que por Resolución n 530 SC/2000 son de cumplimiento obligatorio, deberán ser adaptados por los titulares de las estaciones radioeléctricas involucradas a fin de cumplir con las condiciones impuestas por dichas resoluciones[...]"18.

El control de cumplimiento de los límites de generación pautados, se encuentra a cargo de la Comisión Nacional de Comunicaciones (CNC), en tanto que los gobiernos locales tienen la potestad de autorizar y de ubicar territorialmente la instalación de agentes generadores, determinando los requisitos necesarios para lograr la habilitación ${ }^{19}$.

En el caso que nos ocupa, la localidad de Lanús (Provincia de Buenos Aires) mediante las Ordenanzas n ${ }^{\circ} 9187 / 00$ y n ${ }^{\circ} 9438 / 01$ pone de manifiesto la "posible dañosidad" para la salud derivada de la instalación de antenas de telefonía móvil en su territorio.

En base a estas normas, el intendente de la mencionada localidad dicta, el 30 de noviembre de 2004, el Decreto ${ }^{\circ} 1750$ que dispuso la clausura preventiva y la desactivación de las antenas de telefonía instaladas en Lanús.

${ }^{18}$ En la Ley sobre Telefonía Celular de la Provincia de Santa Fe establece entre los requisitos para la habilitación de antenas la presentación de “...Un informe que contenga el cálculo de la densidad de potencia que aproximadamente emitirá la antena a instalar. Dicho informe de cálculo, deberá ser compatible con los estándares establecidos por las normas nacionales vigentes - Resolución n ${ }^{\circ}$ 202/95 del Ministerio de Salud y Acción Social de la Nación y Resolución n ${ }^{\circ}$ 530/2000 de la Secretaría de Comunicaciones de la Nación, o los parámetros que a futuro determinen los organismos anteriormente mencionados, o los que tuvieren competencia en el tema, acorde a los lineamientos establecidos por organismos competentes internacionales...”. (Art. 2 Ley 12.362/2004)

${ }^{19}$ En el caso de la Provincia de Santa Fe, observamos que, si bien la Ley de Telefonía Celular plasma algunas pautas de ubicación territorial, también otorga potestad a municipios y comunas para tornar más estrictas las prohibiciones y limitaciones: “...La presente ley establece las normas a las que deberán ajustarse los elementos técnicos necesarios para transmisión de comunicaciones y las instalaciones complementarias de telefonía móvil con sus diferentes tipos de soportes y estructuras, como así también los sistemas de enlace troncal (trunking), sin perjuicio de las mayores exigencias que en el ámbito de sus jurisdicciones podrán fijar los Municipios y Comunas, pudiendo definir incluso la prohibición de su instalación en todo el distrito o en determinadas áreas del mismo..." (Art. 1 Ley 12.362/04 Provincia de Santa Fe). 


\subsection{Sobre la causa judicial, condiciones de posibilidad de una "resistencia local"}

Frente al accionar del municipio, se plantea una acción de inconstitucionalidad por parte de la empresa Telefónica Comunicaciones Personales S.A. contra la Municipalidad de Lanús, "Telefónica Comunicaciones Personales S.A. c/ Municipalidad de Lanús s/ acción declarativa de inconstitucionalidad", que fue resuelto en fecha 28.06.2007 por la Sala I de la Cámara Federal de Apelaciones de La Plata.

La empresa plantea la inconstitucionalidad de las disposiciones del Decreto 1750/04 - que retoma el contenido de las Ordenanzas no 9187/00 y 9438/01 - mediante el cual el Intendente de la Municipalidad de Lanús dispuso clausurar preventivamente y desactivar las antenas de telefonía celular móvil existentes, en ejercicio de su poder de policía en materia de salubridad pública, “...las ordenanzas a las que refiere el decreto, por un lado reglamentaron las condiciones y pautas con que deben ser instaladas las antenas en cuestión, a los fines que resulten ambientalmente aptas y, por el otro, prohibieron la instalación de nuevas antenas, suspendiéndose la tramitación de los pedidos de habilitación existentes hasta tanto se efectúen los respectivos estudios y se determine la inocuidad de los campos electromagnéticos generados por dichas antenas..." ${ }^{20}$

En la primera instancia judicial, “...se hizo lugar a la medida cautelar solicitada ordenando a la Municipalidad de Lanús que se abstenga de aplicar a Telefónica Comunicaciones Personales las disposiciones del Decreto $n^{\circ}$ 1750/04 y que proceda al inmediato levantamiento de la clausura y desactivación que ya se hubieren efectivizado sobre la antenas localizadas en jurisdicción de la comuna, emplazadas en las estaciones base pertenecientes a la actora o que utilice mediante acuerdos de colaboración empresaria con Telecom. Personal S.A o Telefónica Argentina S.A...."21.

\footnotetext{
${ }^{20}$ Fragmento de la sentencia "Telefónica Comunicaciones Personales S.A. c/ Municipalidad de Lanús s/ acción declarativa de inconstitucionalidad", ARGENTINA. Cámara Federal de Apelaciones de La Plata. Sentencia de la Cámara Federal de Apelaciones de La Plata, Sala I, Telefónica Comunicaciones Personales SA c/ Municipalidad de Lanús s/ acción declarativa de insconstitucionalidad. La Plata, 28 de junio de 2007. Disponible en: <www. eldiariojudicial.com.ar>. Acceso en: marzo 2009

${ }^{21}$ ARGENTINA. Cámara Federal de Apelaciones de La Plata. Sentencia de la Cámara Federal de Apelaciones de La Plata, Sala I, Telefónica Comunicaciones Personales SA c/ Municipalidad de Lanús s/ acción declarativa de insconstitucionalidad. La Plata, 28 de junio de 2007. Disponible en: <www.eldiariojudicial.com.ar>. Acceso en: marzo 2009
} 
Lo que logra la empresa en la primera instancia judicial es que la Municipalidad de la ciudad de Lanús se vea obligada a dar marcha atrás con su decisión de clausura o desactivación respecto de las antenas que se ubican en su territorio.

Esta resolución fue apelada y en la segunda instancia se modifica el decisorio. Aquí el eje de la discusión se vincula con el alcance y límites de la autonomía municipal cuando se trata de proteger la salud humana y el medioambiente ${ }^{22}$, destacándose que se está ante una hipótesis cuyas consecuencias perjudiciales son controvertidas. Es decir, se trata de un caso que requiere ser pensado dentro de los parámetros mediante los que se delinea la racionalidad precautoria.

De hecho, el planteo escruta si el principio de precaución se enhebraría - y de qué modo - dentro del pull de herramientas que permitirían fundamentar prohibiciones y regulaciones por parte del ente municipal a la luz de la arquitectura de competencias previstas desde la reforma de la Constitución Nacional en 1994. Ésta prevé en su artículo el artículo 123 que "Cada provincia dicta su propia constitución, conforme a lo dispuesto por el artículo $5^{23}$ asegurando la autonomía municipal y reglando su alcance y contenido en el orden institucional, político, administrativo, económico y financiero".

En la discusión sobre el alcance de la autonomía local se podría intentar construir una respuesta que particularice, a la vez, si el hecho de la controversia o incerteza presentarían alguna injerencia especial.

Lo controversial o incierto, se traduce en el campo del derecho en el principio de precaución, inserto en el "microsistema ambiental" ${ }^{24}$. En el texto

${ }^{22}$ La problemática de las competencias de los municipios en materia de gestión de riesgos en general está siendo estudiada por parte de los integrantes del Grupo de Investigación sobre Biorregulaciones (Gonzalo Sozzo, Luciana Sbresso y María Valeria Berros) del Centro de Investigaciones de la Facultad de Ciencias Jurídicas y Sociales de la Universidad Nacional del Litoral.

23 "Cada provincia dictará para sí una constitución bajo el sistema representativo republicano, de acuerdo con los principios, declaraciones y garantías de la Constitución Nacional: y que asegure su administración de justicia, su régimen municipal y la educación primaria. Bajo de estas condiciones el Gobierno federal, garante a cada provincia el goce y ejercicio de sus instituciones" (Art. 5 Constitución Nacional).

${ }^{24}$ La Constitución Nacional prevé en su artículo 41 la determinación de competencias nacionales y provinciales respecto del "problema ambiental", de tal forma que la Nación tiene a su cargo el dictado de leyes de presupuestos mínimos de protección y las provincias las necesarias para complementarlas, sin que ello altere las jurisdicciones locales. Dentro del conjunto de leyes de presupuestos mínimos la Ley 25675/2002 positiviza el principio de precaución como uno de los principios de la política ambiental nacional en su artículo 4 . 
de la sentencia se parte de este "microsistema". Ello se sustentan en la construcción argumental basada en el contenido de la Constitución Nacional (art. 41) y de la "Ley General del Ambiente” (Ley 25.675/2002), “[...] El derecho al ambiente sano exige el ejercicio del deber de preservación que compete a las autoridades ya sean públicas o a las cuales el Estado le ha concesionado prerrogativas de poder público (conf. Art. 41 de la Constitución Nacional) [...]”; "[...] los municipios se encuentran activamente legitimados en cuestiones de incidencia colectiva [...]”; “[...] el Derecho Ambiental requiere de una participación activa de la juricatura, la que si bien de alguna manera pudiera afectar al clásico principio de congruencia, en definitiva se traduce en un obrar preventivo acorde con la naturaleza de los derechos afectados y a la medida de sus requerimientos $[\ldots]^{\prime 25}$

De estos parágrafos transcriptos se extrae la conjugación de dos argumentos relevantes en los que se hace referencia tanto a "lo ambiental" como a lo atinente al contenido de las competencias locales en relación a este tipo de problemáticas. En el primer caso en virtud de los contenidos de derecho ambiental que son traídos por el tribunal a los fines de decidir y que se articulan con el tema competencial.

Asimismo se suma a lo expuesto la estrategia precautoria como herramienta de gestión de riesgos no acabadamente conocidos, todo lo cual da lugar a que la Cámara revoque la resolución apelada. Se dispone que la empresa accionante acompañe un estudio científico detallado sobre la potencial nocividad en la salud pública de los campos electromagnéticos generados por las estaciones de base y tecnologías inalámbricas, además de cumplimentar con la normativa local.

Por contraposición, el voto en disidencia del tribunal, al cual subyace una mirada que podría calificarse como "tolerante" respecto del tema, asigna particular trascendencia al cumplimiento de los estándares por parte de la empresa. Se afirma que “[...] del informe aportado por la Comisión Nacional

\footnotetext{
${ }^{25}$ ARGENTINA. Cámara Federal de Apelaciones de La Plata. Sentencia de la Cámara Federal de Apelaciones de La Plata, Sala I, Telefónica Comunicaciones Personales SA cl Municipalidad de Lanús s/ acción declarativa de insconstitucionalidad. La Plata, 28 de junio de 2007. Disponible en: <www.eldiariojudicial.com.ar>. Acceso en: marzo 2009.
} 
de Comunicaciones surge que al realizar mediciones del nivel de radiaciones electromagnéticas no ionizantes en todas las estaciones de telefonía móvil no se observaron valores encima de los límites establecidos por el Ministerio de Salud y Acción Social establecidos en la Resolución 202/95, no surgiendo acreditado el riesgo para la salud [...]"26. Es decir que, desde la perspectiva de este juez en su voto, el cumplimiento de los niveles aceptados sería óbice para la acción desarrollada por el municipio. Ello se refuerza en que la Comisión Nacional de Comunicaciones es la que tiene la competencia de controlar este aspecto y no los ámbitos locales que sólo regulan lo atinente a su localización.

Ahora bien, el interrogante sería cuál es la extensión y los límites de estas competencias "locales" para determinar "ubicaciones en el territorio".

¿Puede un municipio prohibir la instalación de antenas de telefonía celular u ordenar desactivarlas?

¿Este tipo de competencias presenta alguna connotación específica frente a hipótesis de riesgos controvertidos?

¿Lo controversial tendría alguna injerencia especial en cuanto a profundizar o aminorar las potestades locales en los canales de decisión respecto de "como vivir" en relación a determinados supuestos de hecho que son percibidos socialmente como riesgosos?

En la sentencia, se considera que:

“[...] el régimen municipal que los constituyentes reconocieron como esencial base de la organización política argentina, al consagrarlo como requisito de la autonomía provincial (art. 5 de la Const. Nac.) consiste en la administración de aquellas materias que conciernen únicamente a los habitantes de un distrito o lugar particular sin que afecte directamente a la Nación en su conjunto y, por lo tanto, debe estar investido de la capacidad necesaria para fijar las normas de buena vecindad, ornato, higiene, vialidad, moralidad, etc de la comuna y del poder

${ }^{26}$ ARGENTINA. Cámara Federal de Apelaciones de La Plata. Sentencia de la Cámara Federal de Apelaciones de La Plata, Sala I, Telefónica Comunicaciones Personales SA c/ Municipalidad de Lanús s/ acción declarativa de insconstitucionalidad. La Plata, 28 de junio de 2007. Disponible en: <www.eldiariojudicial.com.ar >. Acceso en: marzo 2009. 
de preceptuar sanciones correccionales para las infracciones de las mismas [... $]^{\prime 27}$.

Esta afirmación puede traducirse en la pregunta acerca de cuáles son las materias que conciernen únicamente a los habitantes de un distrito y cómo ello podría entrar en diálogo con cuestiones relativas a la salud humana y al ambiente en que se desarrolla la cotidianeidad de ese grupo social.

De un análisis de la sentencia se advierte la necesidad de ahondar sobre las potestades de los municipios en las materias mencionadas. Del propio discurso judicial la perspectiva que se construye ensambla ambos planos. Se conjugan expresiones que permiten colocar el tratamiento del supuesto de hecho en la intersección entre "lo ambiental" y "lo relativo a la salud humana". Se refiere, como se afirmó precedentemente, al "microsistema ambiental" y se lo ensambla con fundamentos relacionados con el cuidado de la salud humana bajo la lógica de las competencias locales sobre salubridad pública, estableciéndose que "[...] se advierte que la medida cautelar dictada pretende impedir que la Municipalidad ejerza su legítimo poder de policía en materia de salubridad pública - de conformidad con los arts. 5 y 123 de la Const. Nac; 190 y 192 de la Const. Pcia Bs As; y 26,27 y 226 de la Ley Orgánica de las Municipalidades n 6769/58-, exponiéndose seriamente a la población del distrito a sufrir daños a su salud [...]"28.

Este caso, que llegó a ser objeto de una "estrategia litigial” iniciada por la empresa afectada por la decisión municipal, pone de manifiesto varias cuestiones relevantes.

En primer término, introduce en la agenda la necesidad de determinar cuáles son los alcances de la autonomía municipal en materia medioambiental y

\footnotetext{
${ }^{27}$ ARGENTINA. Cámara Federal de Apelaciones de La Plata. Sentencia de la Cámara Federal de Apelaciones de La Plata, Sala I, Telefónica Comunicaciones Personales SA c/ Municipalidad de Lanús s/ acción declarativa de insconstitucionalidad. La Plata, 28 de junio de 2007. Disponible en: <www.eldiariojudicial.com.ar >. Acceso en: marzo 2009.

${ }^{28}$ ARGENTINA. Cámara Federal de Apelaciones de La Plata. Sentencia de la Cámara Federal de Apelaciones de La Plata, Sala I, Telefónica Comunicaciones Personales SA c/ Municipalidad de Lanús s/ acción declarativa de insconstitucionalidad. La Plata, 28 de junio de 2007. Disponible en: <www.eldiariojudicial.com.ar>. Acceso en: marzo 2009.
} 
relativa a la salud humana a partir del esquema instaurado por el artículo $41^{29}$ de la Constitución Nacional que desde 1994 postula un sistema competencial concurrente, en el cual se "vigorizarían" las potestades locales ${ }^{30}$.

Como se ha afirmado precedentemente, la Nación tiene a su cargo el dictado de leyes de presupuestos mínimos de protección. Las provincias pueden reglamentarlas o complementarlas sin alterar "el piso" de la ley nacional, pudiendo establecer criterios más estrictos. En ambos casos, no se pueden alterar las jurisdicciones locales.

Ello permite construir preguntas acerca de: I) el contenido de las competencias que no pueden ser alteradas por parte de los planos provincial y nacional. En este supuesto, especialmente, aquellas referidas a la gestión de riesgos ambientales y relativos a la salud humana; II) los límites y fundamentos de los criterios "más estrictos" que podrían ser materializados por las autoridades locales en cuanto a la instalación de antenas de telefonía celular; III) las condiciones respecto de la razonabilidad de las medidas: ¿La prohibición o desactivación de antenas de telefonía celular puede ser considerada irrazonable o desproporcionada? Es decir, ¿Cómo opera la relación entre principio precautorio y principio de proporcionalidad ${ }^{31}$ ? ¿Cómo se realiza el juicio de razonabilidad a los efectos de tomar la decisión?

${ }^{29}$ Néstor Cafferatta sostiene que "[...] El artículo 41 luego de la reforma a la Constitución Nacional, en nada restringe la administración de los intereses y servicios locales que les compete a los municipios y comunas sino que, por el contrario, más bien se desprende el fortalecimiento de la autonomía municipal tal como surge en forma expresa del nuevo art. 123 [...]" CAFFERATTA, Nestor. Municipio y medioambiente en la provincia de Buenos Aires. [S.1.]: LLBA, 2007.

${ }^{30}$ MARCHIARO, Enrique. El derecho municipal como derecho posmoderno Argentina. Buenos Aires. Ediar. 2006.

${ }^{31}$ El principio de precaución en Argentina es positivizado incorporando en el mismo concepto este límite al referir a la injerencia de los costos en su aplicabilidad, "Cuando haya peligro de daño grave o irreversible la ausencia de información o certeza científica no deberá utilizarse como razón para postergar la adopción de medidas eficaces, en función de los costos, para impedir la degradación del medio ambiente." ARGENTINA. Leyes nacionales: Ley de Política Ambiental No 25675 - 27 de noviembre de 2002. Disponible en $<$ www.infoleg.gov.ar>. Fecha de acceso: junio 2007. 


\section{Un tercer estadio, algunas conclusiones que permiten delinear tópicos de una agenda en proceso de construcción}

El caso recorrido aparece relevante ya que el "foco de la discusión", que versa sobre la constitucionalidad de una medida local por cuestiones competenciales, permite plantear una serie de interrogantes interesantes.

En principio coadyuva a analizar de qué modo se configura el diálogo entre "lo global" y "lo local" y cuáles son los indicios que se visualizan en las esquematizaciones de una lógica de gestión de riesgos que tienda a estructurarse en uno u otro sentido.

Se trabaja la estandarización del riesgo como manifestación del proceso de globalización respecto del campo jurídico en una problemática cuya particularidad es la controversia científica en que se inscribe.

La estandarización es una estrategia para la gestión de riesgos que ha sido ampliamente extendida en diferentes ámbitos, muchos de los cuales se relacionan con el problema ambiental, disponiendo que el cumplimiento de valores fijados es el límite por debajo del cual no habría causación de daños.

La extensión de esta manera de gobernar riesgos se ha ampliado a numerosas problemáticas, dentro de las cuales algunas de ellas revisten claros rasgos de controversia o incertidumbre científica. Si este tipo de medidas son generalmente tomadas respecto de riesgos percibíos o pensados como "conocidos" o "ciertos", el interrogante podría versar sobre la existencia o no de modificaciones en los razonamientos y argumentos ante supuestos de hecho a los que no subyace esa característica.

El caso analizado permite introducir esta disquisición debido a que frente a riesgos que aparecen como controvertidos se tomó una decisión prohibitiva aunque los estándares fueran los aceptados institucionalmente.

Esta decisión se sustenta en la autonomía local para la toma de decisiones respecto de aquellas problemáticas que se encuentras inescindiblemente ligadas a lo cotidiano. Pone en crisis los límites y pautas de distribución competencial, dado que permite interrogarse sobre la posibilidad que posee una localidad para prohibir totalmente la actividad de instalación de antenas de telefonía móvil, aún en los supuestos en que cumplimenten con los valores de emisión, aspecto cuyo control recae sobre el ámbito nacional. 
Desde una mirada más amplia, y enfocando en la problemática del riesgo, si se considera la perspectiva que sostiene que los riesgos son construidos socialmente $^{32}$ la preguntar a formular podría ser ¿de qué manera desde el derecho se podrían traducir aquellas percepciones, representaciones sociales localizadas espacio - temporalmente?

Este interrogante conduce a analizar de qué modo las vivencias locales son insertas y escuchadas en los espacios de toma de decisiones relativos a riesgos.

En supuestos controversiales o inciertos como el recorrido, en los que el expertise científico ha perdido su "legitimidad moderna" ${ }^{33}$ ¿otras formas de producción de conocimiento y otros saberes, que fueron en paralelo opacados, no postulan la necesaria "recreación" de espacios de diálogo y decisión acerca del "ambiente en el cual vivir" y "vivir sanamente"?

Cuando se piensa en la gestión de riesgos se sostiene que “[...] no puede simplemente evaluarse y gestionarse desde la autoridad con la ayuda de los expertos, sino que debe ser "gobernado" mediante un sistema participativo, dialógico y en el cual la agenda se construya desde la base[...]”34. Entonces, si la decisión municipal hubiera sido el resultado de un proceso ampliamente participativo y dialógico, ¿ello reforzaría el decisorio judicial adoptado? En otras palabras, ¿qué valor tendría la participación ciudadana en este tipo de situaciones?

${ }^{32}$ Existen diferentes concepciones en los estudios sociales sobre riesgos que podrían ser resumidos en dos grandes líneas generales. La primera de ellas, posicionada en una postura epistemológica cognitivista que entiende el riesgo como algo que existe ontológicamente. La segunda, en cambio, parte de concebir a los riesgos como un producto sociocultural y presenta diversos matices en cuanto al cómo es que se construyen socialmente los riesgos. Así, se ubican diferentes puntos de vista: el constructivista fuerte de Mary Douglas; la tesis de la "sociología del riesgo" adonde se posicionan Ulrich Beck y Anthony Giddens como constructivistas "débiles"; la tesis de los governmentality studies de raigrambre neofoucoultiana en la que se encuentra la obra de Francois Ewald, Pat O’Malley y los estudios sociales de la ciencia; y la tesis que concibe el riesgo a modo de concepto. SOZZO, Gonzalo (Coord.). El gobierno de los riesgos. Santa Fé: Universidad Nacional del Litoral, 2007.

${ }^{33}$ SANTOS, Boaventura de Sousa. Crítica de la razón indolente: contra el desperdicio de la experienci. Bilbao: Desclée de Brouwer, 2003.

${ }^{34}$ SOZZO, Gonzalo. Lecciones de Gualeguaychú: de la resistencia a riesgo hacia la construcción de un gobierno alternativo de riesgos ambientale. Revista de la Facultad de Ciencias Jurídicas y Sociales, Santa Fe, n. 5, 2007. Ediciones UNL. 
A manera de conclusión, podría afirmarse que este "caso testigo" da cuenta de las condiciones de posibilidad y de rigidez para la resistencia a algunas estrategias de gestión de riesgos que ponen en cuestión un pull de tecnologías jurídicas de gobierno así como, también, inducen una necesaria revisión de cuestiones vinculadas al ámbito de "lo jurídico" que adquieren especial relevancia para delinear la posibilidad y potencialidad de estrategias de "resistencia local" en relación al problema abordado.

En otras palabras, permite plantear algunos de los tópicos en base a los cuales construir una agenda no cerrada sino susceptible de ser repensada y revisada conforme las diferentes experiencias que puedan recolectarse de la yuxtaposición de supuestos de hecho similares.

\section{Local resistance, the case of installation of cellular antennas in dialogue with risk management strategies}

\section{Abstract}

One of the mechanisms for manages risks related to environment and human health is the establishment of standards or minimum of tolerance, constituted as legal technologies that account the emergence, expansion and development of the precautionary rationality.In the case of the determination of emission limits for non-ionizing radiation from mobile telephony, we see a global strategy of risk management that can be presented as a contribution into the discussion about the globalization process of law. We use this thesis, to recreate, now, what are the strategies that are built locally in "opposition" with this global strategy. This analysis will be done from a study of an individual case and the legal strategies built to articulate the resistance to the installation of these agents release, even in cases that the emission limits are authorized.

Keywords: Risk. Resistance. Precautionary rationality. 


\section{Referências}

ARGENTINA. Cámara Civil y Comercial de Santa Fé. Acción de amparo. Demanda, contestación de demanda, sentencia de primera instancia y sentencia de la Cámara Civil y Comercial de Santa Fe, Sala $2^{\circ}$.Farina, Pablo M. c/ Compañía de Radiocomunicaciones Móviles S.A. s/ Acción de amparo (Zeuz, Tomo 89 - J 360 y siguientes). Santa Fé, 4 de mayo de 2001.

ARGENTINA. Cámara de Apelaciones en lo Civil y Comercial de Rosário. Sentencia de la Cámara de Apelaciones en lo Civil y Comercial de Rosario - Sala I, Vecinos de la Ciudad de Rosario c/ Municipalidad de Rosario. Rosário, 05 de octubre de 2004. LLLitoral Abril 2005, 298.

ARGENTINA. Cámara de Apelaciones en lo Civil y Comercial de Rosário. Sentencia de la Cámara de Apelaciones en lo Civil y Comercial de Rosario Sala I, Vecinos de la Ciudad de Rosario c/ Municipalidad de Rosário. Rosário, 26 de diciembre de 2005. LLLitoral Mayo 2006, 524.

ARGENTINA. Cámara Federal de Apelaciones de La Plata. Sentencia de la Causa 3801/02 de La, Sala II, Asociación Coordinadora de Usuarios, Consumidores $y$ Contribuyentes c/ ENRE - EDESUR s/ cese de obra de cableado y traslado de Subestación Transformadora. Córdoba, 8 de agosto de 2003. Disponíble en: <www. lexisnexis.com.ar> Acceso en: abril 2009.

ARGENTINA. Cámara Federal de Apelaciones de La Plata. Sentencia de la Cámara Federal de Apelaciones de La Plata, Sala I, Telefónica Comunicaciones Personales SA c/ Municipalidad de Lanús s/ acción declarativa de insconstitucionalidad. La Plata, 28 de junio de 2007. Disponible en: <www.eldiariojudicial.com.ar>. Acceso en: marzo 2009.

ARGENTINA. Cámara Federal de La Plata. Sentencia de la Cámara Federal de La Plata - Sala I, Edesur S.A. c/ Municipalidad de Berazategui. La Plata, 30 de agosto de 2007. Disponible en: <www.lexisnexisonline>. Acceso en: abril 2009.

ARGENTINA. Leyes nacionales: ley de política ambiental $n{ }^{\circ} 25675,27$ de noviembre de 2002. Disponible em: <www.infoleg.gov.ar>. Acceso en: junio 2007.

ARGENTINA. Leyes y decretos provinciales: ley de telefonía celular $n^{\circ}$ 12.362/2004 de la Provincia de Santa Fe. Disponible en: <www.santafe.gov.ar $>$. Acceso en: junio 2007.

ARGENTINA. Medida Cautelar. Sentencia En primera instancia del Juzgado en lo Contencioso, Administrativo y Tributario Número 12 de la Ciudad Autónoma de 
Buenos Aires. "Peino, Leonardo Esteban y otros contra GCBA sobre amparo (art.14 $C C A B A)$. Buenos Aires, 18 de octubre de 2006. Disponible en: <www.diariojudicial. com.ar>. Acceso en: marzo 2009.

ARGENTINA. Ordenanzas municipales: Ordenanza no 10578/2000 de la ciudad de Santa Fe. Disponible em: <www.santafeciudad.gov.ar>. Acceso en: Febrero 2009.

ARGENTINA. Sentencia de la Cámara de Apelaciones en lo Contencioso, Administrativo y Tributario - Sala II, Gallo, Susana Beatriz c/ GCBA s/ Amparo (art. 14 CCABA) Causa $n^{\circ}$ 25208/0. Ciudad Autónoma de Buenos Aires, 22 de noviembre de 2007. Disponible en: <www.eldiariojudicial.com.ar>. Acceso en: Marzo 2009.

ARGENTINA. Sentencia de la Cámara en lo Civil y Comercial de la ciudad de Córdoba, no 5, Asís, María F. c/ Giorda Mario y otro s/ daño temido. Córdoba, 20 de marzo de 2007. Disponible en: <www.lexisnexisonline> Acceso en: Abril 2009.

ARGENTINA. Sentencia de la Cámara Federal de Apelaciones de La Plata - Sala III, Agüero, Norberto y otros c/ Municipalidad de Cañuelas s/ amparo. La Plata, 25 de octubre de 2007. Disponible em: <www.eldiariojudicial.com.ar>. Acceso en: Marzo 2009.

ARGENTINA. Sentencia de la Cámara Nacional de Apelaciones en lo Civil de la Ciudad Autónoma de Buenos Aires - Sala H, Bottero, Enrique y otros c/ Nextel Communications Argentina SA s/ daños y perjuicios. Buenos Aires, 5 de diciembre de 2007. Disponible en: <www.lexisnexisonline.com.ar>. Acceso en: Abril 2009.

ARGENTINA. Sentencia del Juzgado en lo Contencioso, Administrativo y Tributario $n^{\circ} 12$ de la Ciudad Autónoma de Buenos Aires, Uyemas Héctor c/ Gobierno de la Ciudad Autónoma de Buenos Aires s/ amparo. Buenos Aires, 18 de septiembre de 2007. Disponible en: <www.eldiariojudicial.com,.ar>. Acceso en: Marzo 2009.

ARGENTINA. Sentencia en causa 17008/03 del Juzgado de Primera Instancia en lo Civil $n{ }^{\circ} 105$ de Buenos Aires. Espíndola María c/ Movicom Bellsouth s/ Daños Perjuicios. Buenos Aires, 09 de febrero de 2007. Disponible en: <www.diariojudicial. com.ar>. Acceso en: Marzo 2009.

ARGENTINA. Tribunal Superior de Córdoba. Sentencia de Castellani, Carlos y E. y otros (Responsabilidad Civil y Seguros). Buenos Aires, 11 de março de 2003, v. 6, n. 6, nov./dic. 2003.

BECK, Ulrich. La sociedad del riesgo globa: siglo XXI. Espana: Paidos, 2002. 
BECK, Ulrich. La sociedad del riesgo mundial. Espana: Paidos, 2007.

BECK, Ulrich. La sociedad del riesgo: hacia una nueva modernida. Espana: Paidos, 1998.

BERROS, María Valeria. La estandarización como tecnología precautoria globalizada: el caso de las radiaciones no ionizante. Prismas: direito, políticas públicas e mundializaçao, Brasília, v. 5, n. 2, p. 127-156, jul./dez. 2008. Colección Universitaria UniCEUB. ISSN 1908-3680.

CAFFERATTA, Nestor. Municipio y medioambiente en la provincia de Buenos Aires. Buenos Aires. LLBA, 2007.

DOUGLAS, Mary. La aceptabilidad del riesgo según las ciencias sociale. Buenos Aires: Paidos, 1996.

GIDDENS, Anthony y otros. Las consecuencias perversas de la modernidad. Barcelona: Anthropos, 1996.

LATOUR, Bruno. Nous niavons jamais été modernes. Paris: La Decouverte, 1997.

MARCHIARO, Enrique. El derecho municipal como derecho posmoderno. Buenos Aires: Ediar, 2006.

ORGANIZACION MUNDIAL DE LA SALUD. Legislación modelo para la protección contra campos electromagnéticos: model legislation for electromagnetic fields protection. Lima: INICTEL-UNI, 2007.

ORGANIZACION MUNDIAL DE LA SALUD. Marco para el desarrollo de estándares de CEM basados en la salud: framework for developing health- based EMF standards. Lima: INICTEL-UNI, 2007.

PROHÍBEN la instalación de antenas de telefonía celular en Firmat. Diario La Capital Buenos Aires, 1 jun. 2006.

SANTOS, Boaventura de Sousa. A globalizacao e as ciencias sociais. Sao Paulo: Cortéz, 2002.

SANTOS, Boaventura de Sousa. Crítica de la razón indolente: contra el desperdicio de la experiencia. Bilbao: Desclée de Brouwer, 2003.

SANTOS, Boaventura de Sousa. La globalización del derecho: los nuevos caminos de la regulación y la emancipación. Bogotá: Universidad Nacional de Colômbia, 2002. 
SOZZO, Gonzalo (Coord.). El gobierno de los riesgos. Santa Fé: Universidad Nacional del Litoral, 2007.

SOZZO, Gonzalo. Lecciones de Gualeguaychú: de la resistencia a riesgo hacia la construcción de un gobierno alternativo de riesgos ambientales. Revista de la Facultad de Ciencias Jurídicas y Sociale, Santa Fé, n. 5, 2007. 